\title{
Observing the Self-assembly of Metal-Organic Frameworks by In-Situ Liquid Cell TEM
}

Joseph P. Patterson, ${ }^{1}$ Patricia Abellan, ${ }^{2}$ Mike S. Denny, Jr., ${ }^{1}$ Chiwoo Park, ${ }^{3}$ Nigel D. Browning, ${ }^{2}$ Seth M. Cohen, ${ }^{1}$ James E. Evans ${ }^{2,4}$ and Nathan C. Gianneschi ${ }^{1} *$

${ }^{1}$ Department of Chemistry \& Biochemistry, University of California, San Diego, La Jolla, California, USA, 92093, USA

${ }^{2}$ Fundamental and Computational Sciences Directorate, Pacific Northwest National Laboratory, P.O. Box 999, Richland, WA 99352, USA

${ }^{3}$.Department of Industrial and Manufacturing Engineering, Florida State University, Tallahassee, FL 32306, USA.

${ }^{4}$ Environmental Molecular Science Laboratory, Pacific Northwest National Laboratory, 3335 Innovation Blvd., Richland, WA 99354, USA

Here we report a significant advancement in materials science made possible by the use of liquid cell transmission electron microscopy. Namely, the ability to observe self-assembly of Metal-Organic Frameworks (MOFs) in liquids with nanometer resolution. MOFs were studied by LCTEM in order to understand and demonstrate control over the dynamics and electron beam effects of these complex macromolecular, beam sensitive materials. With this control, we were then able to observe the nucleation and self-assembly of zeolitic imidazole framework 8 (ZIF-8) giving us the ability to extract growth kinetics, particle size distribution, particle morphology and crystal structure from a single experiment.

The ability to observe dynamic self-assembled materials in liquids at the nanoscale is vital to our understanding of synthetic and biological materials. Liquid cell electron microscopy promises to revolutionize our ability to analyze these materials in liquids due to its unmatched spatiotemporal resolution. A key hurdle for analyzing synthetic or biological self-assembled materials by this method has been the ability to control their behavior under the electron beam and in the nanoliter liquid cells. We report the first observation of nucleation and self-assembly of metal-organic-frameworks by LCTEM providing an unprecedented level of information on their formation by a single technique.

This result has far reaching implication as the ability to control and observe these processes for complex macromolecular, beam sensitive materials demonstrates the applicability of this technique for a wide array of soft and biological materials. LCTEM will become essential for our understanding of nanoscale processes for all materials in liquids; however there is current debate in the literature how useful this tool can be for beam sensitive materials like MOFs. Here we demonstrate that with a careful understanding of how the pre-synthesized materials behave in the liquid cell and under electron beam exposure, suitable conditions can be obtained to observe their self-assembly process with minimal beam effects. Using electron diffraction we confirm the same thermodynamic crystal structure is obtained as under bulk synthetic conditions and show for the first time that individual MOF particles growth through a surface reaction limited mechanism. Furthermore, we demonstrate for the first time the ability to control nucleation density in the cell by materials agitation (flow) rather than all previous studies where nucleation of inorganic crystals was controlled by the electron beam. 


\section{References}

[1] de N Jonge, FM Ross, Nature Nanotechnology6, 2011, p. 695.

[2] OM Yaghi et. al., Chemical Reviews, 112, 2012, p. 673.

[3] TJ Woehl et. al. ACS Nano, 10, 2012, 8599
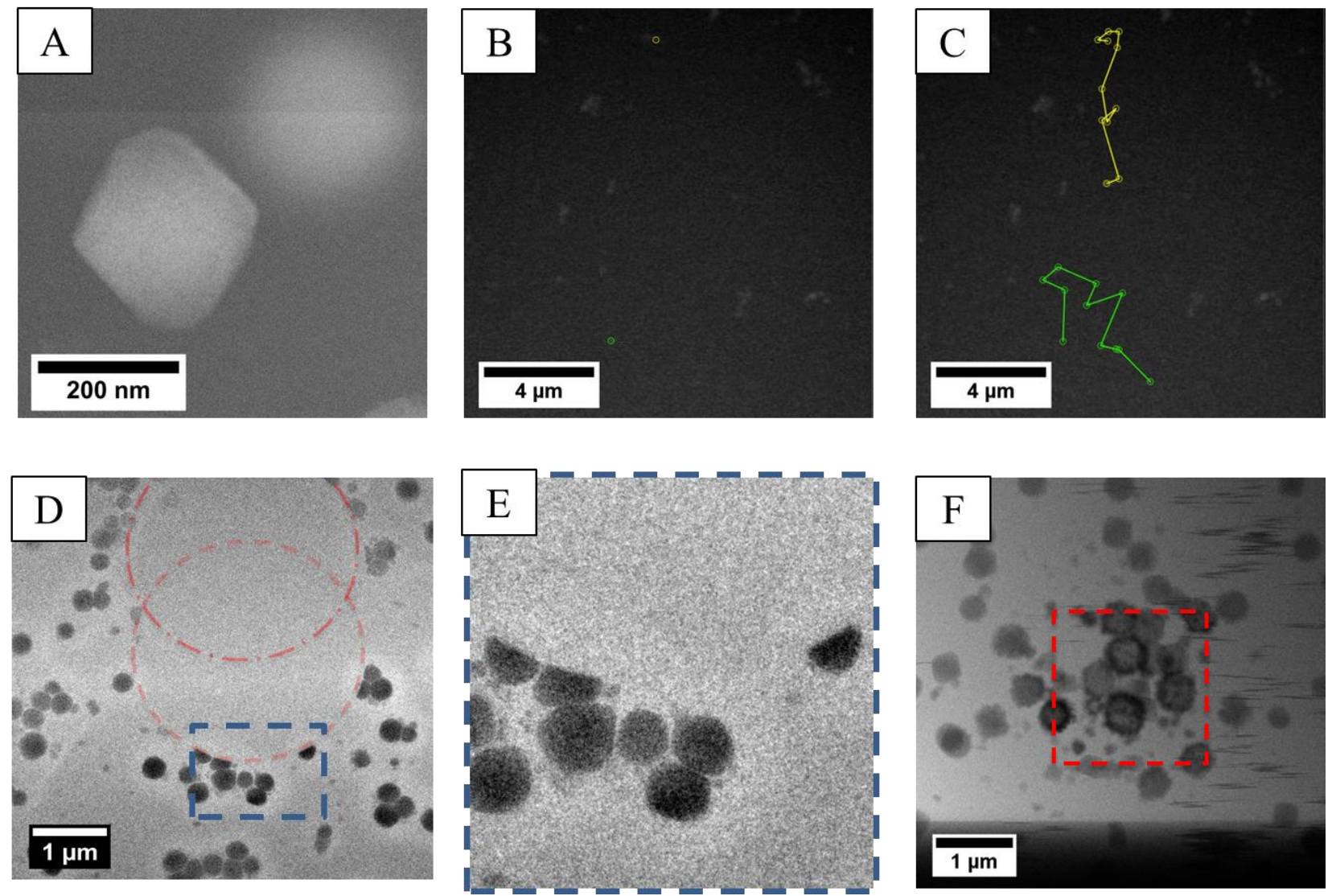

Figure 1. Liquid Cell TEM and STEM images of UiO66 in water. A) high magnification STEM images B) and C) low magnification STEM images tracking particle motion over the period of 6 seconds. D) and E) TEM images after significant beam damage at $300 \mathrm{keV}$ and F) STEM images after significant damage at $80 \mathrm{keV}$.
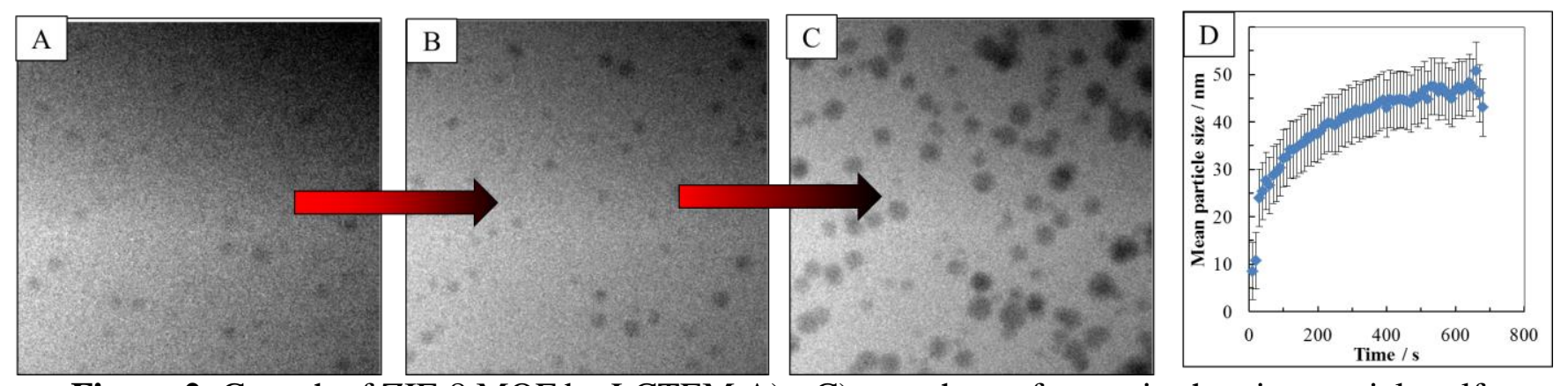

Figure 2. Growth of ZIF-8 MOF by LCTEM A) - C) snapshots of a movie showing particle selfassembly in methanol and D) average growth kinetics extracted directly from movie analysis. 